\title{
Efficacy and Safety of Alirocumab in Individuals with Diabetes Mellitus: Pooled Analyses from Five Placebo-Controlled Phase 3 Studies
}

\author{
Henry N. Ginsberg • Michel Farnier · Jennifer G. Robinson • \\ Christopher P. Cannon - Naveed Sattar - Marie T. Baccara-Dinet • \\ Alexia Letierce · Maja Bujas-Bobanovic · Michael J. Louie · \\ Helen M. Colhoun
}

Received: March 28, 2018 / Published online: May 19, 2018

(C) The Author(s) 2018

\section{ABSTRACT}

Introduction: Diabetes mellitus (DM) carries an elevated risk for cardiovascular disease. Here, we assessed alirocumab efficacy and safety in people with/without DM from five placebo-controlled phase 3 studies.

Methods: Data from up to 78 weeks were analyzed in individuals on maximally tolerated background statin. In three studies, alirocumab

Enhanced digital features To view enhanced digital features for this article go to https://doi.org/10.6084/ m9.figshare.6210182.

Electronic supplementary material The online version of this article (https://doi.org/10.1007/s13300018-0439-8) contains supplementary material, which is available to authorized users.

H. N. Ginsberg ( $\square)$

Columbia University, New York, NY, USA

e-mail: hng1@cumc.columbia.edu

\section{Farnier}

Point Medical and Department of Cardiology, CHU

Dijon-Bourgogne, Dijon, France

J. G. Robinson

University of Iowa, Iowa City, IA, USA

C. P. Cannon

Harvard Clinical Research Institute, Boston, MA, USA

N. Sattar

University of Glasgow, Glasgow, UK
$75 \mathrm{mg}$ every 2 weeks (Q2W) was increased to $150 \mathrm{mg}$ Q2W at week 12 if week 8 low-density lipoprotein cholesterol (LDL-C) was $\geq 70 \mathrm{mg} /$ $\mathrm{dL}$; two studies used alirocumab $150 \mathrm{mg}$ Q2W throughout. The primary endpoint was percentage change in LDL-C from baseline to week 24.

Results: In the alirocumab $150 \mathrm{mg}$ pool $(n=2416)$, baseline LDL-C levels were $117.4 \mathrm{mg} / \mathrm{dL}(\mathrm{DM})$ and $130.6 \mathrm{mg} / \mathrm{dL}$ (without $\mathrm{DM})$, and in the $75 / 150 \mathrm{mg}$ pool $(n=1043)$ $112.8 \mathrm{mg} / \mathrm{dL}(\mathrm{DM})$ and $133.0 \mathrm{mg} / \mathrm{dL}$ (without $\mathrm{DM})$. In the $150 \mathrm{mg}$ Q2W group, week 24 LDL-C reductions from baseline were observed in persons with DM (-59.9\%; placebo, $-1.4 \%)$ and without DM (-60.6\%; placebo, $+1.5 \%) ; 77.7 \%$ (DM) and $76.8 \%$ (without DM) of subjects achieved LDL-C $<70 \mathrm{mg} / \mathrm{dL}$. In the alirocumab 75/150 mg group, 26\% (DM) and 36\% (without

M. T. Baccara-Dinet

Sanofi, Montpellier, France

A. Letierce

Sanofi, Chilly-Mazarin, France

M. Bujas-Bobanovic

Sanofi, Paris, France

M. J. Louie

Regeneron Pharmaceuticals Inc, Tarrytown, NY, USA

H. M. Colhoun

University of Edinburgh, Edinburgh, UK 
DM) of subjects received dose increase. In this group, week 24 LDL-C levels changed from baseline by $-43.8 \%$ (DM; placebo, $+0.3 \%$ ) and $-49.7 \%$ (without DM; placebo, $+5.1 \%$ ); LDL$\mathrm{C}<70 \mathrm{mg} / \mathrm{dL}$ was achieved by $68.3 \%$ and $65.8 \%$ of individuals, respectively. At week 24 , alirocumab was also associated with improved levels of other lipids. Adverse event rates were generally comparable in all groups (79.8-82.0\%).

Conclusions: Regardless of DM status, alirocumab significantly reduced LDL-C levels; safety was generally similar.

Funding: Sanofi and Regeneron Pharmaceuticals, Inc.

Plain Language Summary: Plain language summary available for this article.

Keywords: Alirocumab; Cholesterol-lowering drugs; Diabetes mellitus; LDL-C; PCSK9

\section{PLAIN LANGUAGE SUMMARY}

High cholesterol puts people at risk of heart disease, especially those with diabetes. Physicians set individualized cholesterol treatment goals for each patient. Statins, prescribed to reduce high cholesterol levels, may not lower cholesterol enough in all people. Alirocumab is a medication for lowering cholesterol levels. Alirocumab is intended for use in combination with maximally tolerated statin. Here we compared the effects of alirocumab to 1054 people with diabetes to 2445 people without diabetes.

Our study showed that most people with and without diabetes reached the cholesterol goal of less than $70 \mathrm{mg}$ per deciliter after 24 weeks of treatment with individualized alirocumab doses. Treatment with alirocumab 150 milligrams every 2 weeks resulted in $78 \%$ of people with diabetes and $77 \%$ of people without diabetes reaching this goal. Treatment with alirocumab $75 \mathrm{mg}$ every 2 weeks (with some individuals getting their dose increased to $150 \mathrm{mg}$ ) also produced similar results in people with diabetes (68\%) and without diabetes $(66 \%)$. Eighty percent of people with diabetes and $80 \%$ of people without diabetes had adverse reactions with alirocumab treatment. Similar rates of adverse reactions were reported in the corresponding placebo groups with diabetes $(82 \%)$ and without diabetes (81\%). Regardless of diabetes status, the most common adverse reactions among alirocumab-treated people were common cold, chest infection, and injection-site reaction.

In summary, alirocumab provides an additional treatment option for people with and without diabetes who do not to reach their cholesterol goals, even with maximally tolerated statin dose.

\section{INTRODUCTION}

Cardiovascular disease (CVD) is the most common cause of death in persons with diabetes mellitus (DM) $[1,2]$. Individuals with DM are, on average, at double the risk of atherosclerotic CVD (ASCVD) in comparison to those without DM, and the presence of dyslipidemia in people with type $2 \mathrm{DM}$ further increases the risk of adverse cardiovascular outcomes [3, 4].

The elevated cardiovascular risk associated with DM is recognized in guidelines $[1,3,5,6]$, which recommend more intense management strategies for low-density lipoprotein cholesterol (LDL-C)-lowering in individuals with DM than for the general population. Statins are recommended as first-line therapy to reduce LDL-C in DM $[1,5,7,8]$. However, many people with DM have persistent lipid abnormalities despite statin treatment $[9,10]$. The 2017 updated American College of Cardiology Expert Consensus Task Force and the 2018 American Diabetes Association standards of care recommend that a proprotein convertase subtilisin/ kexin type 9 (PCSK9) inhibitor be considered in individuals with ASCVD and DM whose LDL-C levels are not optimally controlled on high-intensity statin therapy $[6,8]$.

The 2017 update of the European Society of Cardiology/European Atherosclerosis Society Task Force also recommends the use of a PCSK9 inhibitor for two categories of individuals with DM, depending on LDL-C levels: (1) individuals with DM and clinical ASCVD whose LDL-C 
levels are $>100 \mathrm{mg} / \mathrm{dL}$ and (2) individuals with $\mathrm{DM}$ and familial hypercholesterolemia without clinical ASCVD whose LDL-C levels are $>140 \mathrm{mg} / \mathrm{dL}$, despite maximally tolerated statin and ezetimibe therapies [11].

The lipid profile of type $2 \mathrm{DM}$ is characterized by reduced high-density lipoprotein cholesterol (HDL-C), high triglycerides, and an increase in the proportion of LDL-C particles that are small and dense, with or without high levels of LDL-C $[12,13]$. In the ODYSSEY DMINSULIN study (NCT02585778), alirocumab $75 \mathrm{mg}$ every 2 weeks (Q2W; with possible dose increase to $150 \mathrm{mg}$ Q2W) significantly reduced LDL-C levels and other lipids in individuals with type $1 \mathrm{DM}(n=76)$ or type $2 \mathrm{DM}(n=441)$ treated with insulin [14]. Furthermore, in individuals with type $2 \mathrm{DM}$ and mixed dyslipidemia, the same alirocumab dosing regimen also resulted in significant reductions in nonHDL-C, LDL-C, and other lipids in ODYSSEY DM-DYSLIPIDEMIA (NCT02642159) [15]. However, the efficacy and safety of alirocumab $75 \mathrm{mg}$ Q2W (with possible dose adjustment to $150 \mathrm{mg}$ Q2W) and $150 \mathrm{mg}$ Q2W (without dose adjustment) have not been compared in a larger pool of individuals with and without DM treated for longer duration. This subgroup analysis of five placebo-controlled phase 3 studies (LONG TERM [NCT01507831] [16], HIGH FH [NCT01617655] [17], COMBO I [NCT01644175] [18], FH I [NCT01623115] [19], and FH II [NCT01709500] [19]) aimed to compare the efficacy and safety of alirocumab in a large group of individuals with and without DM at baseline, with the primary efficacy endpoint being LDL-C reduction from baseline to week 24 .

\section{METHODS}

\section{Trial Participants and Study Designs}

The current analysis used patient-level data from study participants according to alirocumab dosing regimen who enrolled for five double-blind, randomized, placebo-controlled ODYSSEY phase 3 studies with 52-78 weeks' treatment duration. Methods for each of the individual studies have been previously reported [16-19].

In summary, all participants, with or without DM, had hypercholesterolemia at study entry and were on maximally tolerated, stable, background statin therapy with or without other lipid-lowering agents. The FH I and FH II studies recruited participants with heterozygous familial hypercholesterolemia $(\mathrm{HeFH})$ and at either very high risk (LDL-C $\geq 70 \mathrm{mg} / \mathrm{dL}$ with prior CVD) or high risk (LDL-C $\geq 100 \mathrm{mg} / \mathrm{dL}$ but no prior CVD). The HIGH FH study enrolled individuals with $\mathrm{HeFH}$ and LDL-C levels $\geq 160 \mathrm{mg}$ / dL. The LONG TERM study included participants who either had HeFH or established coronary heart disease (CHD) or CHD risk equivalents based on the European Systematic Coronary Risk Estimation (SCORE), with baseline LDL-C $\geq 70 \mathrm{mg} / \mathrm{dL}$. The COMBO I study included participants with either established CVD and $\mathrm{LDL}-\mathrm{C} \geq 70 \mathrm{mg} / \mathrm{dL}$, or CHD risk equivalents and LDL-C $\geq 100 \mathrm{mg} / \mathrm{dL}$, based on the European SCORE, which included DM with other risk factors or chronic kidney disease.

All study protocols were approved by the appropriate institutional review boards, and all participants provided informed, written consent. All trials were performed in accordance with the ethical principles that have their origin in the Declaration of Helsinki and all applicable amendments laid down by the World Medical Assemblies and the International Conference Harmonisation Guidelines for Good Clinical Practice.

Participants were randomized to alirocumab or placebo groups in a 2:1 ratio. Two studies (LONG TERM and HIGHFH) used an alirocumab dose of $150 \mathrm{mg}$ Q2W throughout the trial period. The other three studies (COMBO I, FH I, and FH II) used an initial alirocumab dose of $75 \mathrm{mg}$ Q2W, with an increase to $150 \mathrm{mg}$ Q2W at week 12 if the LDL-C level at week 8 remained $\geq 70 \mathrm{mg} / \mathrm{dL}$. All doses were delivered by subcutaneous injection (alirocumab $75 \mathrm{mg}$, alirocumab $150 \mathrm{mg}$, or placebo) using a 1-mL dose volume.

Participants were classified as having DM (type 1 or 2 ) or not, according to medical history reported by the investigator. 


\section{Efficacy Analysis}

Efficacy was compared in individuals with and without DM in two pools according to alirocumab dose regimen (pool of $150 \mathrm{mg}$ Q2W studies; pool of $75 \mathrm{mg}$ Q2W with possible increase to $150 \mathrm{mg}$ Q2W, abbreviated in the text to $75 / 150 \mathrm{mg} \mathrm{Q} 2 \mathrm{~W}$ ).

The main efficacy endpoint for this pooled analysis was the mean percentage change in calculated LDL-C from baseline to week 24 (the primary endpoint of the individual studies). Other efficacy endpoints included percentage change in calculated LDL-C from baseline to week 12 (prior to potential dose increase in trials utilizing the 75/150 mg Q2W dosing regimen), proportion of individuals achieving riskbased LDL-C goals, and the percentage change in other lipid parameters from baseline to weeks 12 and 24 . Mean percentage change in LDL-C and other lipid parameters over time is also reported up to week 78 .

Changes from baseline for the main LDL-C efficacy endpoint and other lipid values were statistically evaluated with an intention-to-treat (ITT) approach, which included lipid data from all randomized persons regardless of adherence to treatment. The analysis utilized a mixed-effect model with repeated measures to account for missing data, as previously described [20]. Data on changes over time were presented according to ITT analysis, and on-treatment analysis using a modified ITT approach, which included only lipid data collected while the individual was receiving study treatment.

Other lipid parameters were analyzed either in the same way as the main efficacy endpoint or, in the case of lipoprotein (a) $[\operatorname{Lp}(\mathrm{a})]$ and triglyceride (TG) percentage changes, and LDLC goal achievement proportions, analysis involved a multiple imputation approach then robust regression (for $\mathrm{Lp}(\mathrm{a})$ and TG percentage changes) or logistic regression (for LDL-C goal achievement), in the ITT population.

Consistency of treatment effect across subgroups was assessed by providing interaction $p$ values. A further subgroup analysis was performed to compare alirocumab efficacy in persons with and without DM according to HeFH status at week 12 and week 24 .

\section{Safety Analysis}

Safety data are reported in subgroups of individuals assigned to alirocumab or placebo, regardless of alirocumab dose, according to baseline DM status. All adverse events, regardless of seriousness and irrespective of potential relationship to alirocumab, were recorded by the investigators up to the last visit planned in the protocol. Treatment-emergent adverse events (TEAEs) were defined as events that developed, worsened, or became serious between the first and last dose of study treatment plus 70 days, classified according to the Medical Dictionary for Regulatory Activities. Adverse events of special interest included injection-site reactions, general allergic events, neurocognitive disorders, and adjudicated cardiovascular events. Statistical analysis of the safety population included all randomized individuals who received at least one dose or part of a dose of study drug, and safety data were analyzed by descriptive statistics.

The effect of alirocumab treatment on glycated hemoglobin (HbA1c) and fasting plasma glucose (FPG) was also evaluated according to DM status throughout the studies using descriptive statistics conducted on the safety population.

\section{RESULTS}

\section{Study Participants}

In total, $30.1 \%$ of alirocumab-treated individuals $(n=699)$ and $30.2 \%$ of those receiving placebo $(n=355)$ were classified as having DM at baseline ( $n=1625$ and $n=820$, respectively, were classified as not having diabetes) (Table 1 ; Supplementary Fig. 1). In total, 24 persons $(0.69 \%)$ had type $1 \mathrm{DM}$ and the remainder had type $2 \mathrm{DM}$. Individuals with DM were generally older and had a higher BMI versus those without DM. Fewer subjects with DM were male, white, or had HeFH compared with subjects without DM (Table 1). Fewer participants with DM had a history of ASCVD (62.5-62.8\%) compared with those without DM (72.4-74.6\%). Regardless of DM status, all 
Table 1 Baseline demographics, clinical characteristics, and lipid profile in individuals with and without DM (randomized population)

\begin{tabular}{|c|c|c|c|c|}
\hline & \multicolumn{2}{|c|}{ Individuals with $\mathrm{DM}(n=1054)$} & \multicolumn{2}{|c|}{ Individuals without DM $(n=2445)$} \\
\hline & $\begin{array}{l}\text { Alirocumab } \\
(n=699)\end{array}$ & $\begin{array}{l}\text { Placebo } \\
(n=355)\end{array}$ & $\begin{array}{l}\text { Alirocumab } \\
(n=1625)\end{array}$ & $\begin{array}{l}\text { Placebo } \\
(n=820)\end{array}$ \\
\hline Age, years, mean $(\mathrm{SD})$ & $61.7(9.5)$ & $60.8(10.2)$ & $57.3(12.2)$ & $57.9(11.7)$ \\
\hline Male, $n(\%)$ & $405(57.9)$ & $192(54.1)$ & $1010(62.2)$ & $520(63.4)$ \\
\hline Race, white, $n$ (\%) & $581(83.1)$ & $290(81.7)$ & $1558(95.9)$ & $782(95.4)$ \\
\hline BMI, $\mathrm{kg} / \mathrm{m}^{2}$, mean $(\mathrm{SD})$ & $32.4(6.3)$ & $32.9(6.0)$ & $29.1(5.0)$ & $29.2(5.1)$ \\
\hline $\mathrm{ASCVD}^{\mathrm{a}}, n(\%)$ & $439(62.8)$ & $222(62.5)$ & $1176(72.4)$ & $612(74.6)$ \\
\hline $\mathrm{CHD}, n(\%)$ & $388(55.5)$ & $193(54.4)$ & $1066(65.6)$ & $573(69.9)$ \\
\hline $\mathrm{ACS}, n(\%)$ & $247(35.3)$ & $134(37.7)$ & $733(45.1)$ & $394(48.0)$ \\
\hline $\begin{array}{l}\text { Coronary revascularization } \\
\text { procedure, } n(\%)\end{array}$ & $271(38.8)$ & $133(37.5)$ & $735(45.2)$ & $389(47.4)$ \\
\hline $\begin{array}{l}\text { Other clinically significant } \\
\mathrm{CHD}^{\mathrm{b}}, n(\%)\end{array}$ & $145(20.7)$ & $74(20.8)$ & $477(29.4)$ & $248(30.2)$ \\
\hline Peripheral arterial disease, $n(\%)$ & $32(4.6)$ & $23(6.5)$ & $65(4.0)$ & $33(4.0)$ \\
\hline Ischemic stroke, $n(\%)$ & $60(8.6)$ & $26(7.3)$ & $139(8.6)$ & $60(7.3)$ \\
\hline $\mathrm{HeFH}, n(\%)$ & $85(12.2)$ & $55(15.5)$ & $753(46.3)$ & $364(44.4)$ \\
\hline High-intensity $\operatorname{statin}^{\mathrm{c}}, n(\%)$ & $311(44.5)$ & $153(43.1)$ & $1016(62.5)$ & $529(64.5)$ \\
\hline With $\mathrm{HeFH}$ & $63(74.1)$ & $42(76.4)$ & $600(79.7)$ & $294(80.8)$ \\
\hline Without $\mathrm{HeFH}$ & $248(40.4)$ & $111(37.0)$ & $416(47.7)$ & $235(51.5)$ \\
\hline With ASCVD & $213(48.5)$ & $115(51.8)$ & $697(59.3)$ & $377(61.6)$ \\
\hline Without ASCVD & $98(37.7)$ & $38(28.6)$ & $319(71.0)$ & $152(73.1)$ \\
\hline \multicolumn{5}{|l|}{ Baseline lipids, mean (SD), mg/dL } \\
\hline Calculated LDL-C & $116.5(37.6)$ & $119.7(41.2)$ & $131.3(48.9)$ & $129.8(45.9)$ \\
\hline Non-HDL-C & $150.0(42.6)$ & $151.6(46.7)$ & $158.1(52.1)$ & $157.2(49.2)$ \\
\hline Apo B & $101.6(26.4)$ & $101.1(28.0)$ & $105.4(30.0)$ & $105.2(28.7)$ \\
\hline Lp(a), median (Q1, Q3) & $21.1(6.0,58.0)$ & $19.0(5.8,61.6)$ & $26.0(10.0,73.0)$ & $24.9(7.5,71.8)$ \\
\hline Fasting TGs, median (Q1, Q3) & $147.0(108.0,205.3)$ & $144.0(105.3,205.0)$ & $118.0(85.0,163.7)$ & $120.7(88.0,169.0)$ \\
\hline
\end{tabular}


Table 1 continued

\begin{tabular}{lllllll}
\hline & \multicolumn{2}{l}{ Individuals with DM $(n=1054)$} & & \multicolumn{2}{l}{ Individuals without DM $(n=2445)$} \\
\cline { 2 - 3 } & $\begin{array}{l}\text { Alirocumab } \\
(n=699)\end{array}$ & $\begin{array}{l}\text { Placebo } \\
(n=355)\end{array}$ & & $\begin{array}{l}\text { Alirocumab } \\
(\boldsymbol{n}=1625)\end{array}$ & $\begin{array}{l}\text { Placebo } \\
(\boldsymbol{n}=\mathbf{8 2 0})\end{array}$ \\
\hline HDL-C & $47.6(11.6)$ & $48.1(12.3)$ & & $51.1(13.8)$ & $50.5(13.2)$ \\
\hline
\end{tabular}

$A C S$ acute coronary syndrome, Apo apolipoprotein, $A S C V D$ atherosclerotic cardiovascular disease, $B M I$ body mass index, $C H D$ coronary heart disease, $D M$ diabetes mellitus, $H D L-C$ high-density lipoprotein cholesterol, $\mathrm{HeFH}$ heterozygous familial hypercholesterolemia, $L D L-C$ low-density lipoprotein cholesterol, $L p(a)$ lipoprotein (a), Q1, Q3 first and third quartiles, $S D$ standard deviation, $T G$ triglyceride

${ }^{a}$ Included CHD, peripheral arterial disease, and ischemic stroke; for study FH II, ischemic stroke, transient ischemic attack, carotid endarterectomy, or carotid artery stent procedure and renal artery stent procedure were also included

b Diagnosed by invasive or non-invasive testing

c High-intensity statins defined as atorvastatin $40-80 \mathrm{mg}$, rosuvastatin $20-40 \mathrm{mg}$, or simvastatin $80 \mathrm{mg}$

patients received statin therapy. Baseline highintensity statin use was lower among individuals with DM (43.1-44.5\%) versus without DM (62.5-64.5\%), but was greater in individuals with $\mathrm{HeFH}$ versus those without (74.1-80.8\% vs $37.0-51.5 \%$, respectively). In total, $48.6-61.6 \%$ of individuals with ASCVD and $28.6-73.1 \%$ of those without ASCVD received high-intensity statin at baseline (Table 1).

Overall, individuals with DM had lower baseline Lp(a) and HDL-C levels but higher TG levels than individuals without DM (Table 1).

Among individuals with DM, 27.8\% were receiving injectable treatments which always consisted of insulin (27.8\%), sometimes combined with a glucagon-like peptide 1 antagonist (GLP 1; 3.3\%). No individuals were receiving GLP 1 antagonist only.

\section{Efficacy}

\section{LDL-C Outcomes: Pool of Alirocumab $150 \mathrm{mg}$ Q2W Studies (LONG TERM and HIGH FH)}

At week 24 in the full alirocumab $150 \mathrm{mg}$ Q2W cohort, the least-squares mean LDL-C levels were changed from baseline by $-59.9 \%$ (with DM; placebo, $-1.4 \%$ ) and $-60.6 \%$ (without DM; placebo, $+1.5 \%$ ) (Table 2 ). Regardless of high-intensity statin status, the LDL-C percentage change from baseline to week 24 was similar in participants with and without DM (Fig. 1). Mean week 24 LDL-C levels of $52.3 \mathrm{mg} /$ $\mathrm{dL}$ were achieved in alirocumab-treated individuals with DM (absolute change from baseline, $-73.6 \mathrm{mg} / \mathrm{dL}$ ) and $50.2 \mathrm{mg} / \mathrm{dL}$ in those without DM (absolute change from baseline, $-75.7 \mathrm{mg} / \mathrm{dL}$ ). LDL-C levels $<70 \mathrm{mg} / \mathrm{dL}$ at week 24 were attained by $77.7 \%$ of alirocumabtreated individuals with DM and $76.8 \%$ of those without DM (placebo, 10.3\% and 6.4\%, respectively) (Table 2).

In the same alirocumab $150 \mathrm{mg}$ Q2W group, least-squares mean percentage change from baseline to week 12 in LDL-C levels was $-62.1 \%$ in individuals with DM (placebo, $+0.1 \%)$ and $-62.9 \%$ in individuals without DM (placebo, $+1.7 \%$ ) (Supplementary Table 1 ). At week 12, alirocumab-associated LDL-C changes were similar for patients with or without DM in the HeFH subgroup (DM, $-56.2 \%$; non-DM, $-57.2 \%$ ) and the non-HeFH subgroup (DM, - 62.5\%; non-DM, - 65.4\%) (Supplementary Tables 2 and 3). LDL-C levels were maintained through 78 weeks in individuals with and without DM in the on-treatment population (Fig. 2a). The reductions up to 78 weeks for the ITT population are presented in Supplementary Fig. 2A.

In the HeFH subjects from the LONG TERM and HIGH FH studies, least-squares mean LDL-C levels changed from baseline to week 24 by 


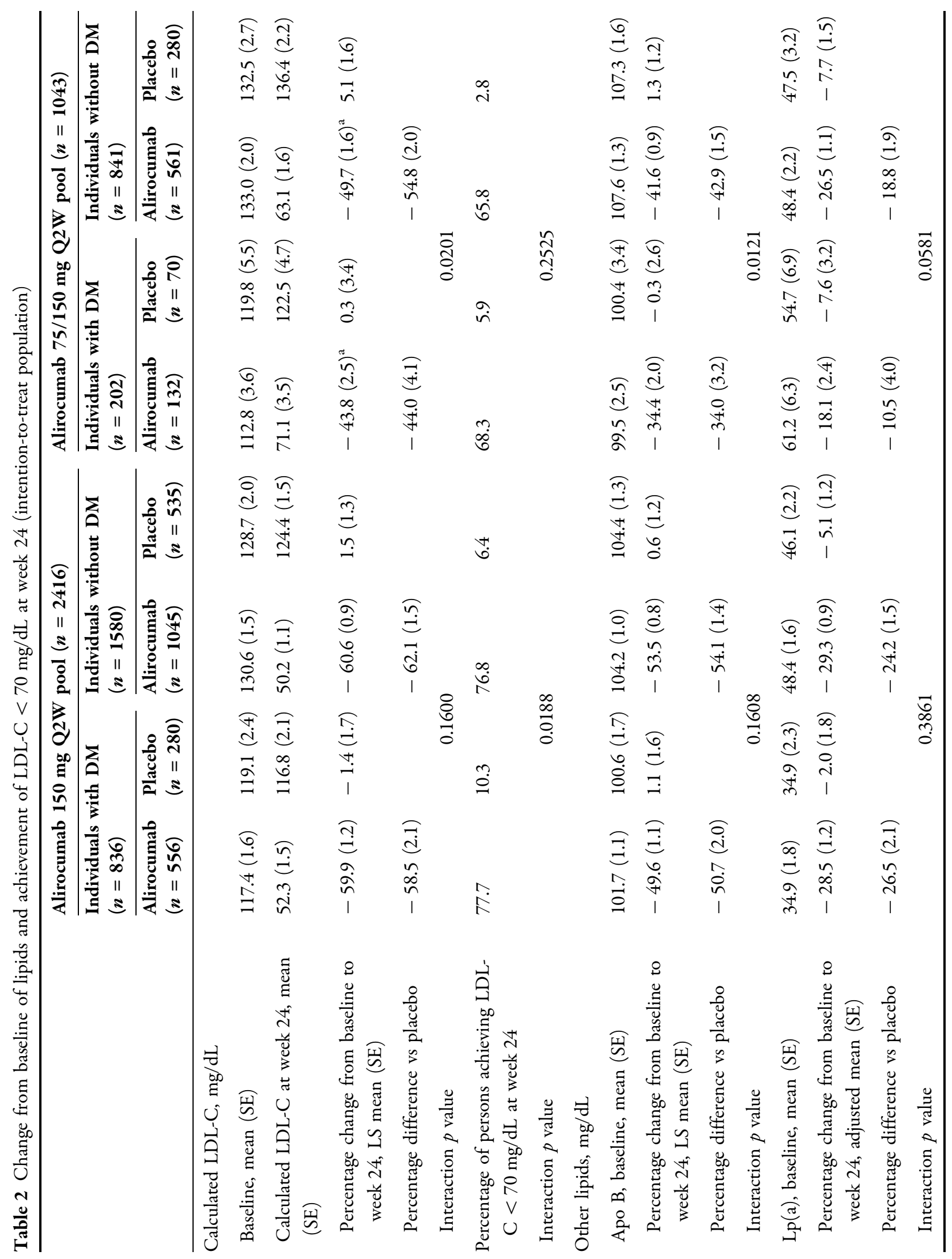




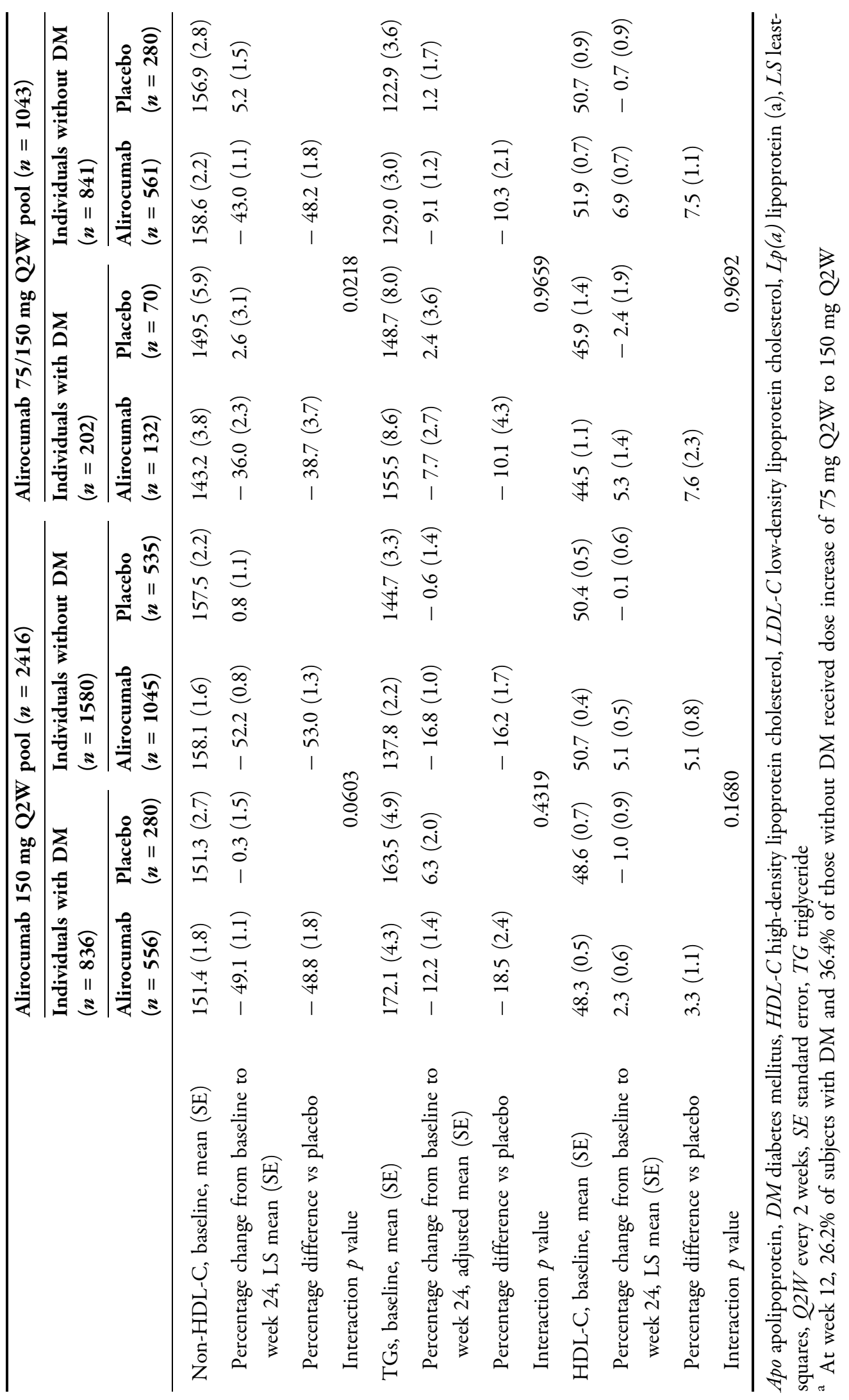




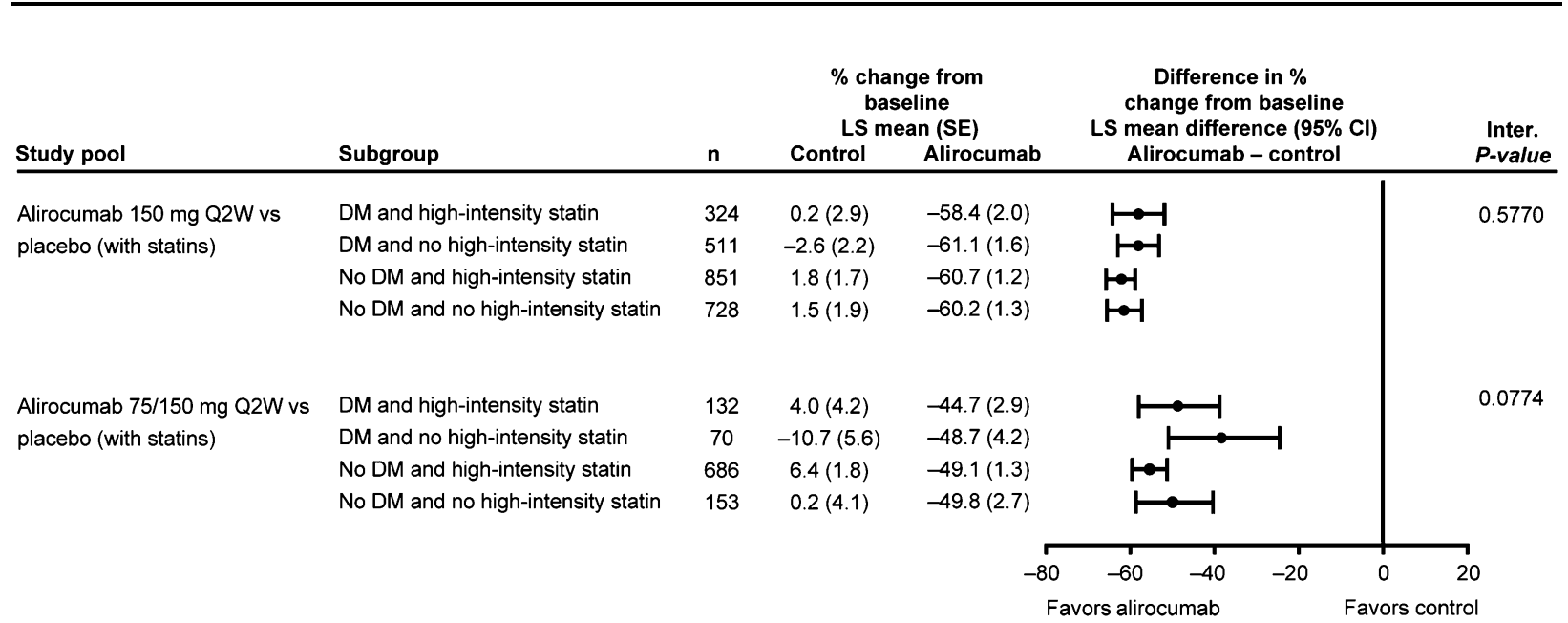

Fig. 1 Percentage change from baseline in calculated LDL-C at week 24-subgroup analysis by DM status and statin intensity at baseline (intention-to-treat population). High-intensity statin defined as atorvastatin $40-80 \mathrm{mg}$, rosuvastatin $20-40 \mathrm{mg}$, or simvastatin $80 \mathrm{mg}$.

$-53.1 \%$ (with DM; placebo, $+0.1 \%$ ) and $-55.3 \%$ (without DM; placebo, + 1.5\%) (Supplementary Table 4). In subjects without HeFH, the least-squares mean LDL-C changes from baseline to week 24 were $-60.3 \%$ in subjects with DM (placebo, $-1.4 \%$ ) and $-62.9 \%$ in those without DM (placebo, + 1.6\%) (Supplementary Table 5).

\section{LDL-C Outcomes: Pool of Alirocumab 75/150 mg Q2W Studies (FH I, FH II, and COMBO I)}

At week 24 in the full 75/150 mg Q2W cohort, least-squares mean percentage change from baseline in LDL-C level was - 43.8\% (with DM; placebo, $+0.3 \%$ ) and $-49.7 \%$ (without DM; placebo, $+5.1 \%$ ) (Table 2). Mean week 24 LDL$\mathrm{C}$ levels of $71.1 \mathrm{mg} / \mathrm{dL}$ were achieved in alirocumab-treated individuals with DM (absolute change from baseline, $-58.3 \mathrm{mg} / \mathrm{dL}$ ) and $63.1 \mathrm{mg} / \mathrm{dL}$ in those without DM (absolute change from baseline, $-66.3 \mathrm{mg} / \mathrm{dL}$ ). Subgroup analysis with and without high-intensity statin demonstrated similar LDL-C reductions at week 24, regardless of DM status (Fig. 1).

At week 12 in this cohort (i.e., before possible dose increase), least-squares mean percentage change from baseline to week 12 in LDL-C levels was $-38.0 \%$ in persons with DM
$C I$ confidence interval, $D M$ diabetes mellitus, $L D L$ - $C$ lowdensity lipoprotein cholesterol, $L S$ least-squares, $S E$ standard error

(placebo, $+7.0 \%$ ) and $-46.0 \%$ in those without DM (placebo, $+3.4 \%$ ) in the alirocumab 75/150 mg Q2W treatment pool (Supplementary Table 1). When the HeFH and non-HeFH subjects in this cohort were examined separately, similar percentage reductions from baseline to week 12 were observed in alirocumab-treated groups, except for a less pronounced change in LDL-C levels in individuals with DM in the HeFH subgroup (-34.9\%; placebo, $+3.6 \%$ ) (Supplementary Tables 2 and 3).

The protocol for the three studies starting with the alirocumab $75 \mathrm{mg}$ Q2W dose specified a blinded dose increase from $75 \mathrm{mg}$ to $150 \mathrm{mg}$ Q2W at week 12 (if week 8 LDL-C levels were $\geq 70 \mathrm{mg} / \mathrm{dL}$ ); $26.2 \%$ of individuals with $\mathrm{DM}$ received a dose increase to $150 \mathrm{mg} \mathrm{Q} 2 \mathrm{~W}$, as did $36.4 \%$ of those without DM. In subjects remaining on $75 \mathrm{mg}$ Q2W, the baseline LDL-C levels were lower in those with DM $(99.5 \mathrm{mg} /$ $\mathrm{dL})$ versus subjects without DM $(117.9 \mathrm{mg} / \mathrm{dL}$; interaction $p$ value $<0.0001)$. In subjects receiving alirocumab dose increase to $150 \mathrm{mg}$ Q2W, the baseline LDL-C levels were $149.7 \mathrm{mg} /$ $\mathrm{dL}(\mathrm{DM})$ and $159.4 \mathrm{mg} / \mathrm{dL}$ (without DM).

LDL-C reductions were maintained with alirocumab 75/150 mg Q2W until end of study treatment, regardless of DM status, in the ontreatment population (Fig. 2a). Similar LDL-C 

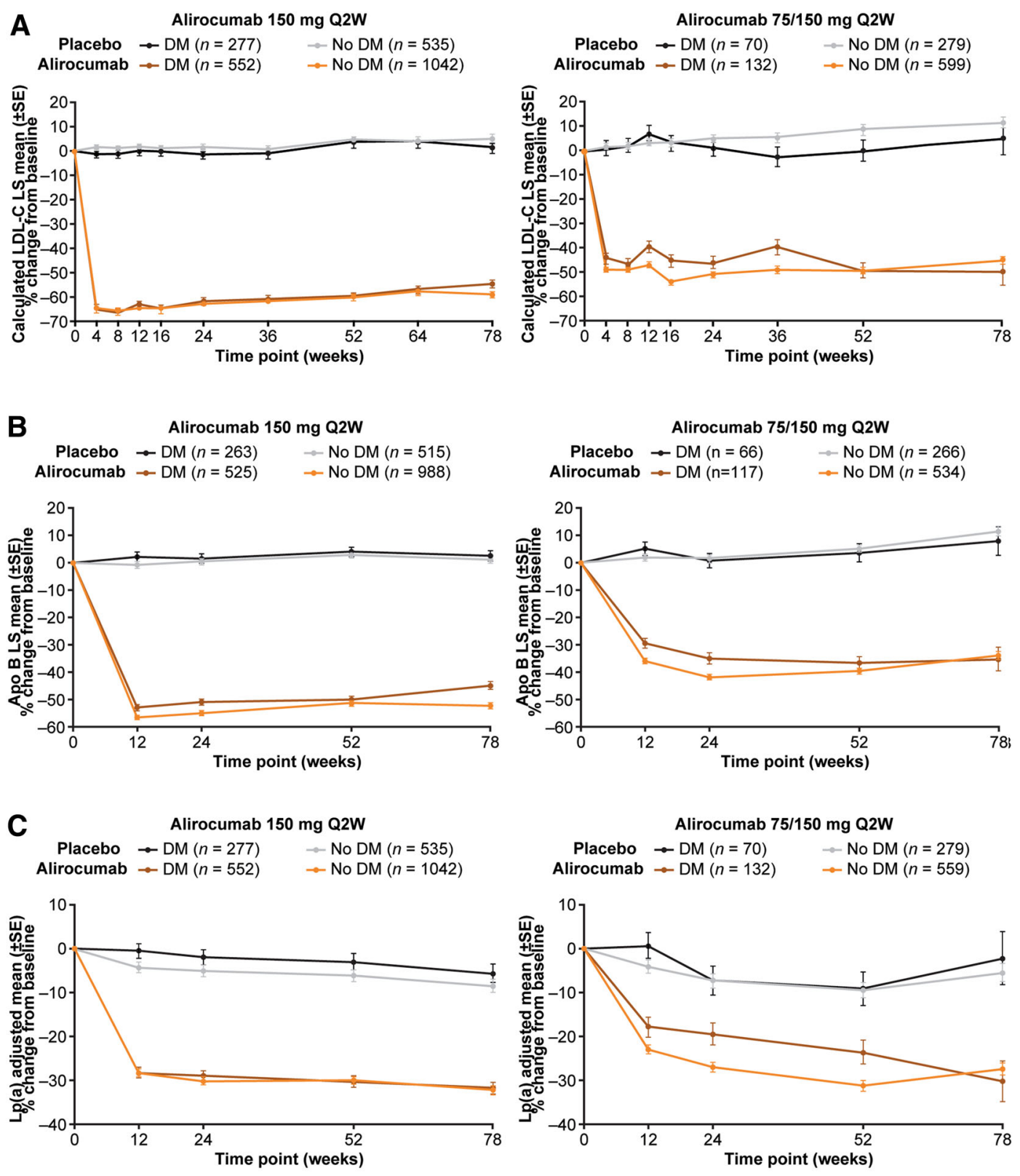

D

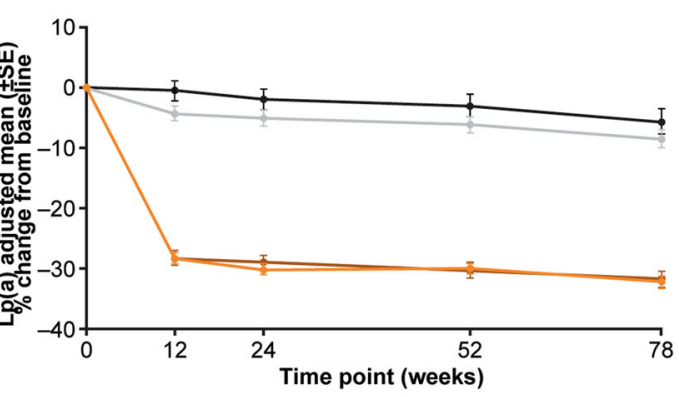

Alirocumab $150 \mathrm{mg}$ Q2W
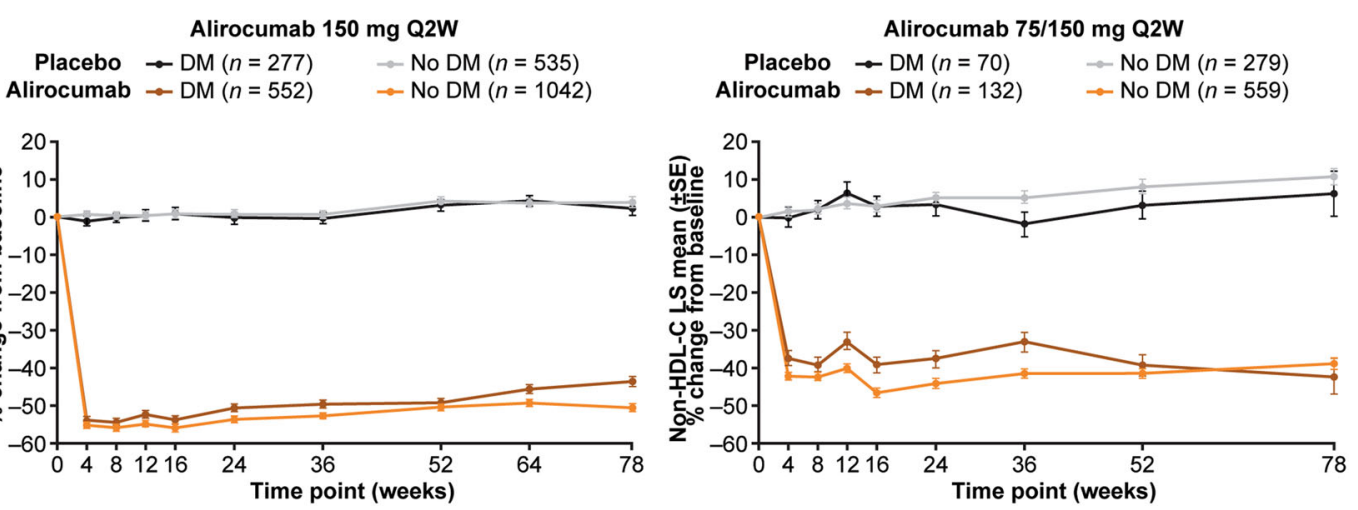
4Fig. 2 Percentage change from baseline over time for a LDL-C, b Apo B, c Lp(a), d non-HDL-C, e TGs, and f HDL-C according to DM status for the alirocumab $150 \mathrm{mg}$ Q2W and $75 / 150 \mathrm{mg}$ Q2W treatment pools (modified intention-to-treat population). Apo apolipoprotein, $D M$ diabetes mellitus, $H D L-C$ high-density lipoprotein cholesterol, $L D L-C$ low-density lipoprotein cholesterol, $L p($ a) lipoprotein (a), $L S$ least-squares, $Q 2 W$ every 2 weeks, $S E$ standard error, $T G$ triglyceride

reductions were observed in the ITT population (Supplementary Fig. 2a).

At week $24, \mathrm{LDL}-\mathrm{C}<70 \mathrm{mg} / \mathrm{dL}$ was attained by $68.3 \%$ of individuals in the alirocumab group with DM and $65.8 \%$ without DM (placebo, $5.9 \%$ and $2.8 \%$, respectively) (Table 2 ).

In the HeFH subgroup, the proportion of individuals requiring dose increase from alirocumab $75 \mathrm{mg}$ to $150 \mathrm{mg}$ Q2W was $48.7 \%$ (DM) and $41.2 \%$ (without DM). At week 24, leastsquares mean percentage changes in LDL-C level were $-52.3 \%$ in persons with DM
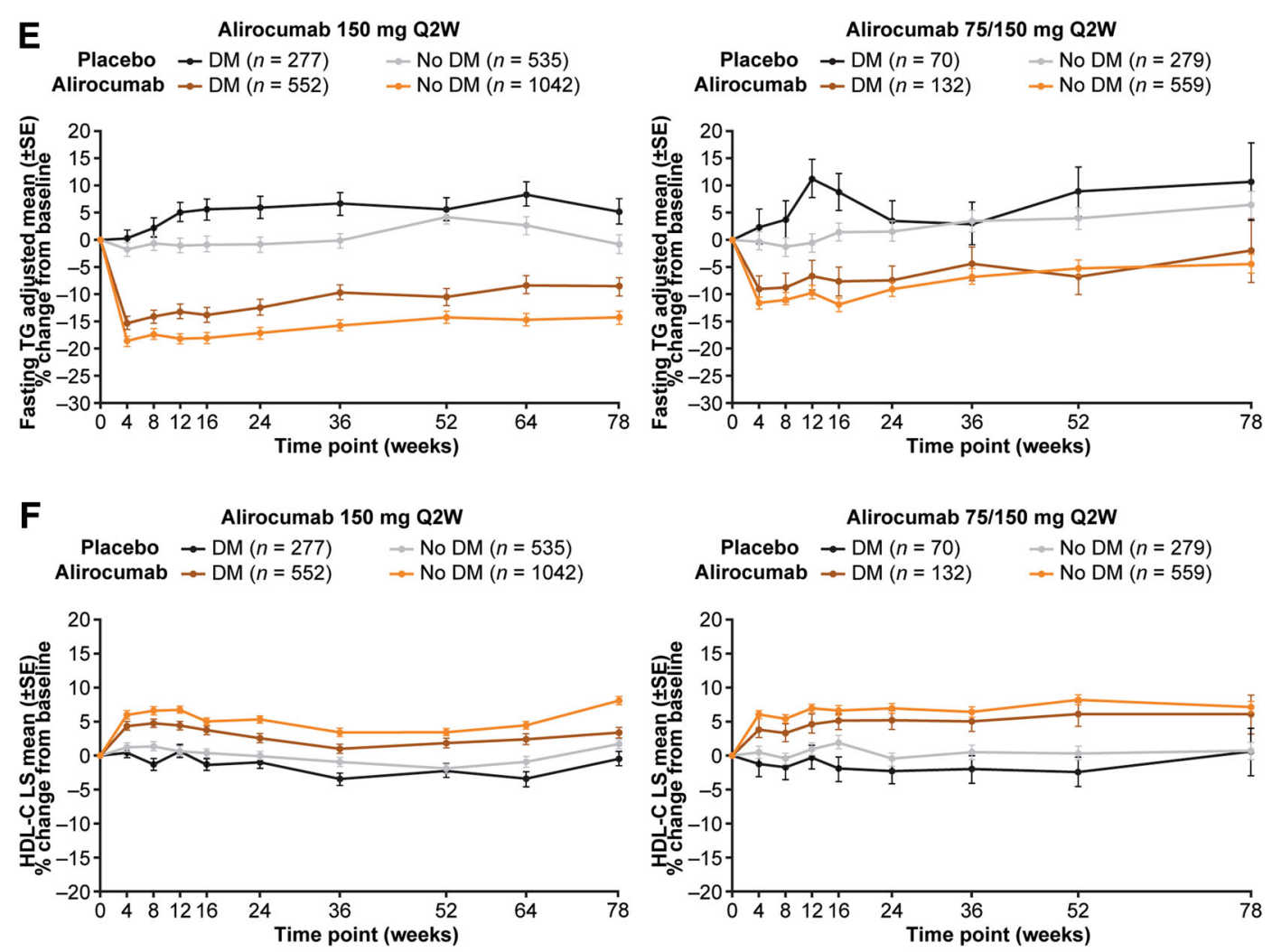

(placebo, $+2.7 \%$ ) and $-48.4 \%$ in those without DM (placebo, +7.6\%) (Supplementary Table 4). In the non-HeFH subgroup, $16.5 \%$ of persons with DM and $17.0 \%$ of those without DM received dose adjustment at week 12 . In this same group, the week 24 least-squares mean LDL-C levels changed from baseline by $-42.2 \%$ in persons with DM (placebo, $-2.6 \%$ ) and $-53.2 \%$ in those without DM in the subgroup (placebo, $-2.0 \%$ ) (Supplementary Table 5).

\section{Other Lipid Parameters}

From baseline to week 24, alirocumab treatment was associated with reduced levels of apolipoprotein (Apo) B, Lp(a), non-HDL-C, and TGs, and increased levels of HDL-C (Table 2). In the alirocumab $150 \mathrm{mg}$ Q2W pool, no differences between subjects with and without DM were observed (Table 2). In the alirocumab 75/150 mg Q2W pool, lower percentage changes in Apo B and non-HDL-C were observed in participants with DM versus those without (interaction $P$ values $<0.05$ ) (Table 2). The

Fig. 2 continued 
percentage changes in TG and HDL-C levels were similar regardless of DM status.

From baseline to week 12, alirocumab dosing regimens of $150 \mathrm{mg}$ Q2W and 75/150 $\mathrm{mg}$ Q2W were associated with improved levels of Apo B, Lp(a), non-HDL-C, TGs, and HDL-C in subjects with or without DM (Supplementary Table 1).

In persons with and without $\mathrm{HeFH}$, similar alirocumab efficacy was observed at week 12 , regardless of DM status and alirocumab dosing regimen, except for lower Apo B and non-HDL$\mathrm{C}$ levels in individuals with $\mathrm{HeFH}$ in the alirocumab 75/150 mg group with DM (interaction $p$ value $<0.05$ between persons with $\mathrm{DM}$ and those without) (Supplementary Tables 2 and 3).

Reduced levels of other lipids were observed from week 4 and maintained for up to 78 weeks in individuals with and without DM in the ontreatment population (Fig. 2b-f). The lipid levels for the ITT population were similar (Supplementary Fig. 2B-F). In the HeFH group, improvements in other lipids from baseline to week 24 were seen in individuals with DM and without, regardless of alirocumab dosing (Supplementary Table 4). No differences between subjects with and without DM were observed in the non-HeFH group either, except for lower mean percentage reductions in Apo B levels in subjects with DM in both alirocumab groups and lower mean percentage reductions in nonHDL-C levels in subjects with DM in the alirocumab $150 \mathrm{mg}$ Q2W pool (interaction $p$ value $<0.05$ between persons with $\mathrm{DM}$ and those without; Supplementary Table 5).

No correlation was found between baseline HbA1c levels and LDL-C reductions in alirocumab-treated individuals with DM at week 24 (Supplementary Fig. 3).

\section{Safety Analysis}

Overall, the incidence of TEAEs was similar between groups, occurring in $79.9 \%$ with DM and $79.8 \%$ without DM in alirocumab-treated individuals versus $82.0 \%$ and $81.0 \%$ in the placebo groups, respectively (Table 3 ). The most common TEAEs occurring in $\geq 5 \%$ of individuals treated with alirocumab were nasopharyngitis, reported in $11.5 \%(n=80)$ of individuals with DM and $13.0 \%(n=211)$ of those without $\mathrm{DM}$, and upper respiratory tract infection, reported in $7.9 \%(n=55)$ and $6.6 \%(n=107)$, respectively. Among alirocumab-treated individuals, $3.7 \%(n=26)$ of those with $\mathrm{DM}$ and $8.7 \%(n=141)$ of individuals without DM reported injection-site reactions (Table 3). Overall, 20.3\% (alirocumab) and 23.9\% (placebo) of subjects in the DM group and $15.0 \%$ (alirocumab) and $14.3 \%$ (placebo) of those without DM experienced treatment-emergent serious adverse events. Discontinuation rates were $5.6 \%$ (alirocumab) and $8.0 \%$ (placebo) in individuals with $\mathrm{DM}$, and $5.4 \%$ (alirocumab) and $5.7 \%$ (placebo) in those without DM (Table 3). Occurrence of adverse events of special interest in alirocumab-treated individuals was generally similar regardless of DM status (Table 3). Overall, the median exposure to randomized treatment was 78 weeks in both the alirocumab and control groups, regardless of DM status. Mean HbA1c and FPG levels remained unchanged in both groups throughout the treatment period regardless of DM status (Supplementary Table 6).

\section{DISCUSSION}

Across all studies in the ODYSSEY program, individuals with hypercholesterolemia experienced significant LDL-C reductions from baseline compared with either placebo or ezetimibe following alirocumab treatment with background statin therapy ( \pm other lipid-lowering therapies) [16-19]. Similar reductions were seen with alirocumab treatment in individuals with or without DM, and were maintained for up to 78 weeks.

In the present analysis, alirocumab $150 \mathrm{mg}$ Q2W resulted in $58.5 \%$ and $62.1 \%$ reductions in LDL-C levels versus placebo at week 24 with no significant difference being observed between those with and those without DM, respectively. Furthermore, LDL-C levels of $<70 \mathrm{mg} / \mathrm{dL}$ were achieved by the majority of persons receiving this dose regardless of DM status (76.9-77.7\%), with mean achieved LDL-C levels of $50.2-52.3 \mathrm{mg} / \mathrm{dL}$, regardless of DM status. 
Table 3 Adverse events in persons with and without DM (safety population)

\begin{tabular}{|c|c|c|c|c|}
\hline \multirow[t]{2}{*}{$n(\%)$} & \multicolumn{2}{|c|}{$\mathrm{DM}(n=1051)$} & \multicolumn{2}{|c|}{ No DM $(n=2441)$} \\
\hline & $\begin{array}{l}\text { Alirocumab } \\
(n=696)\end{array}$ & $\begin{array}{l}\text { Placebo } \\
(n=355)\end{array}$ & $\begin{array}{l}\text { Alirocumab } \\
(n=1622)\end{array}$ & $\begin{array}{l}\text { Placebo } \\
(n=819)\end{array}$ \\
\hline TEAEs & $556(79.9)$ & $291(82.0)$ & $1295(79.8)$ & $663(81.0)$ \\
\hline Treatment-emergent SAEs & $141(20.3)$ & $85(23.9)$ & $244(15.0)$ & $117(14.3)$ \\
\hline $\begin{array}{l}\text { TEAEs leading to } \\
\text { discontinuation }\end{array}$ & $56(8.0)$ & $20(5.6)$ & $88(5.4)$ & $47(5.7)$ \\
\hline TEAEs leading to death & $7(1.0)$ & $5(1.4)$ & $9(0.6)$ & $8(1.0)$ \\
\hline \multicolumn{5}{|l|}{ Adverse events of special interest } \\
\hline HLT: injection site reactions & $26(3.7)$ & $10(2.8)$ & $141(8.7)$ & $52(6.3)$ \\
\hline $\begin{array}{l}\text { General allergic TEAE } \\
(\mathrm{CMQ})\end{array}$ & $63(9.1)$ & $28(7.9)$ & $163(10.0)$ & $77(9.4)$ \\
\hline Neurocognitive disorders & $9(1.3)$ & $6(1.7)$ & $12(0.7)$ & $3(0.4)$ \\
\hline $\begin{array}{l}\text { Adjudicated cardiovascular } \\
\text { events }\end{array}$ & $37(5.3)$ & $27(7.6)$ & $57(3.5)$ & $19(2.3)$ \\
\hline $\mathrm{ALT}>3 \times \mathrm{ULN}, n / N(\%)$ & $11 / 689(1.6)$ & $14 / 349(4.0)$ & $34 / 1610(2.1)$ & $7 / 815(0.9)$ \\
\hline \multicolumn{5}{|c|}{ TEAEs occurring in $\geq 5 \%$ of persons } \\
\hline Nasopharyngitis & $80(11.5)$ & $35(9.9)$ & $211(13.0)$ & $107(13.1)$ \\
\hline Upper respiratory infection & $55(7.9)$ & $36(10.1)$ & $107(6.6)$ & $58(7.1)$ \\
\hline Injection-site reaction & $26(3.7)$ & $10(2.8)$ & $141(8.7)$ & $52(6.3)$ \\
\hline Bronchitis & $33(4.7)$ & $27(7.6)$ & $79(4.9)$ & $31(3.8)$ \\
\hline Urinary tract infection & $49(7.0)$ & $26(7.3)$ & $79(4.9)$ & $39(4.8)$ \\
\hline Arthralgia & $27(3.9)$ & $26(7.3)$ & $91(5.6)$ & $50(6.1)$ \\
\hline Influenza & $39(5.6)$ & $19(5.4)$ & $108(6.7)$ & $44(5.4)$ \\
\hline Back pain & $33(4.7)$ & $17(4.8)$ & $90(5.5)$ & $53(6.5)$ \\
\hline Headache & $29(4.2)$ & $16(4.5)$ & $90(5.5)$ & $48(5.9)$ \\
\hline Diarrhea & $33(4.7)$ & $17(4.8)$ & $90(5.5)$ & $40(4.9)$ \\
\hline Myalgia & $21(3.0)$ & $11(3.1)$ & $90(5.5)$ & $35(4.3)$ \\
\hline
\end{tabular}

ALT alanine aminotransferase, CMQ custom MedDRA query, DM diabetes mellitus, HLT high-level term, MedDRA Medical Dictionary of Regulatory Activities, $S A E$ serious adverse event, TEAE treatment-emergent adverse event, $U L N$ upper limit of normal

In the pool of studies where per-protocol dose increase from $75 \mathrm{mg}$ to $150 \mathrm{mg}$ Q2W occurred if LDL-C goals were not reached at week 8 , the week 24 LDL-C levels from baseline were changed by $-43.8 \%$ (individuals with DM) and $-49.7 \%$ (individuals without DM) in the alirocumab groups (placebo, $+0.3 \%$ and $+5.1 \%$, respectively), with these changes being 
consistent regardless of DM status. Possibly as a result of the higher LDL-C levels at baseline, alirocumab dose was increased at week 12 in a higher proportion of subjects without DM, which was associated with a greater magnitude of LDL-C reduction at week 24 in that group (absolute LDL-C change, $-66.3 \mathrm{mg} / \mathrm{dL}$ ) versus the DM group (absolute LDL-C change, $-58.3 \mathrm{mg} / \mathrm{dL}$ ); however, similar LDL-C levels $(63.1-71.1 \mathrm{mg} / \mathrm{dL})$ were achieved in both groups. In the subgroup without $\mathrm{HeFH}$, the percentage of individuals requiring dose increase was similar regardless of DM status. Therefore, this analysis indicates that the higher proportion of individuals in the subgroup without DM who received alirocumab dose increase versus the subgroup with DM could be explained by more individuals with $\mathrm{HeFH}$ with higher baseline LDL-C being included in the subgroup without DM.

At baseline, the differences in prevalence of $\mathrm{HeFH}, \mathrm{ASCVD}$, and high-intensity statin use, and baseline LDL-C levels between individuals with and without DM may reflect discrepancies in selection criteria between the studies included in the analysis. Furthermore, consistent with previous studies [21, 22], fewer individuals with HeFH had DM (12.2-15.5\%) compared with those without HeFH (44.4-46.3\%). Possibly as a result of this imbalance, fewer individuals in the cohort with DM had a history of ASCVD and received high-intensity statin compared with the cohort without DM. The lower rate of high-intensity statin use in individuals with $\mathrm{DM}$ indicates that this high-risk population is undertreated.

Overall, alirocumab treatment was generally well tolerated with no particular differences observed in incidence of TEAEs, serious adverse effects, or deaths compared with placebo in individuals with or without DM. Overall, mean exposure to randomized treatment was similar (78 weeks) in individuals with or without DM regardless of treatment status. This analysis does not allow for conclusive safety observations to be made, in particular for rare adverse events such as adjudicated cardiovascular events. As a consequence, safety results should be considered in the context of the overall ODYSSEY program.
Fewer injection-site reactions were reported in individuals with DM, a difference that may in part be due to a greater familiarity and tolerance associated with glucometer and/or injectable medication use in the DM population (27.8\% and $72.2 \%$ of persons with DM were receiving antihyperglycemic injectable and non-injectable medication at baseline, respectively). However, as previously reported, administration by injection does not deter persons from self-administration of alirocumab [23]. The ODYSSEY OUTCOMES study (NCT01663402) will help to further establish if DM status has an influence on the frequency of injection-site reactions [24].

Mean HbA1c and FPG measurements in individuals with DM were comparable between alirocumab and placebo for up to 78 weeks of treatment. These variables were comparable between alirocumab and placebo in individuals without DM, as previously reported [25]. On the basis of these results, alirocumab treatment does not appear to affect blood glucose, which is very reassuring given that statins modestly raise the risk of DM [26], and recent genetic publications have suggested that the PCSK9 pathway might be relevant to glycemia levels $[27,28]$. In a subgroup analysis of 11,031 individuals with DM in FOURIER (median follow-up, 2.2 years), neither HbA1c nor incidence of DM was increased in the evolocumab groups versus the placebo groups [29]. In contrast, a recent metaanalysis of phase $2 / 3$ randomized PCSK9 inhibitor clinical studies (excluding FOURIER) suggested that PCSK9-mediated lowering of LDL-C does increase risk for DM [30]. Taking all of these publications into consideration, it is not yet clear whether LDL-C reduction per se or the means of attaining lower LDL-C levels influence DM risk. In the ODYSSEY and FOURIER studies, most individuals received statin therapy, which may mask the glycemic effect of PCSK9 inhibitors $[29,31]$. The results of ODYSSEY OUTCOMES will provide key additional data relevant to this important issue.

Overall efficacy and safety findings from these placebo-controlled studies were consistent with findings from studies comparing alirocumab with ezetimibe and employing background statin therapy $[32,33]$. A recently 
published study of the efficacy and safety of alirocumab in individuals with type $2 \mathrm{DM}$ and mixed dyslipidemia (defined as non-HDL$\mathrm{C} \geq 100 \mathrm{mg} / \mathrm{dL} ; \quad \mathrm{TGs} \geq 150 \mathrm{mg} / \mathrm{dL} \quad$ and $<500 \mathrm{mg} / \mathrm{dL}$ ) showed similar responses for non-HDL-C (37.7\% reduction) and TGs (13.0\% reduction) at week 24 compared to the larger group of individuals with type 1 and $2 \mathrm{DM}$ in the present analysis [15]. In the DM-INSULIN phase $3 b$ study, alirocumab demonstrated similar results in reducing LDL-C and other lipids in insulin-treated type $1 \mathrm{DM}$ and type $2 \mathrm{DM}$ individuals [14]; all individuals were at high cardiovascular risk and received maximally tolerated statin therapy.

This analysis was limited by the non-randomized nature of DM status in subgroups, which could introduce bias to the analyses. Nevertheless, this placebo-controlled analysis of up to 78 weeks adds to the body of evidence on PCSK9 inhibitor use in people with DM [34].

\section{CONCLUSION}

On the basis of these data from placebo-controlled phase 3 studies, DM status does not appear to meaningfully affect lipid-modifying efficacy or safety of alirocumab treatment, nor does alirocumab appear to significantly affect blood glucose control in individuals with or without DM. The results presented here provide support for the recent recommendation by the American Diabetes Association that PCSK9 inhibitor therapy may be considered for individuals with DM and ASCVD [8]. The ODYSSEY OUTCOMES study is expected to provide an opportunity to evaluate the effect of alirocumab in a larger sample of individuals with DM [24].

\section{ACKNOWLEDGEMENTS}

The authors would like to thank study individuals and investigators, and the following persons from sponsors for their contributions to data collection and analysis, assistance with statistical analysis, or critical review of the manuscript: Regeneron Pharmaceuticals Inc.: Eva-Lynne Greene, Carol Hudson, Rita Samuel, and Desmond Thompson; Sanofi: Corinne Hanotin, Michael Howard, L. Veronica Lee, Guillaume Lecorps, Laurence Merlet, Christelle Lorenzato, and Jonas Mandel.

Funding. This analysis as well as journal article processing charges were funded by Sanofi and Regeneron Pharmaceuticals, Inc. The sponsor was involved in the study design, collection, analysis, and interpretation of data, as well as data checking of information provided in the manuscript. The authors had unrestricted access to data, were responsible for all content and editorial decisions, and received no honoraria related to the development of this publication.

Medical Writing and Editorial Assistance. Medical writing support was provided by Susanne Ulm, PhD, and Rachel Wright, PhD, of Prime (Knutsford, UK), funded by Sanofi and Regeneron Pharmaceuticals Inc. according to Good Publication Practice guidelines.

Authorship. All named authors meet the International Committee of Medical Journal Editors (ICMJE) criteria for authorship for this article, take responsibility for the integrity of the work as a whole, and have given their approval for this version to be published.

Author Contributions. Henry N. Ginsberg, Michel Farnier, and Jennifer G. Robinson contributed to study design or concept, data acquisition, analysis and interpretation of the data, and critically reviewed and edited the manuscript. Christopher P. Cannon, Marie T. Baccara-Dinet, Maja Bujas-Bobanovic, Michael J. Louie, and Helen M. Colhoun contributed to study design or concept, analysis and interpretation of the data, and critically reviewed and edited the manuscript. Naveed Sattar and Alexia Letierce contributed to analysis and interpretation of data, and critically reviewed and edited the manuscript.

Disclosures. Henry N. Ginsberg has received other research support from Genzyme (Sanofi), Merck, Sanofi, Regeneron Pharmaceuticals Inc., and Amgen; and is a consultant and on the 
advisory board for Amarin, Amgen, AstraZeneca, Bristol Myers Squibb, GlaxoSmithKline, IONIS, Janssen, Kowa, Merck, Novartis, Sanofi, Regeneron Pharmaceuticals, Inc., and Pfizer. Michel Farnier has received research support from and participated in a speakers' bureau for Amgen, Merck, and Sanofi; received honoraria from Abbott/Mylan, Akcea/Ionis, Eli Lilly, and Pfizer; and acted as a consultant/advisory panel member for Amgen, AstraZeneca, Roche, Kowa, Merck, Pfizer, Sanofi, and Servier. Jennifer G. Robinson has received research grants from Amarin, Amgen, AstraZeneca, Daiichi-Sankyo, Eli Lilly, Esai, Genentech/F. Hoffman La Roche, GlaxoSmith Kline, Merck, Pfizer, Regeneron Pharmaceuticals, Inc./Sanofi, and Zinfandel/ Takeda; and is a consultant and on the advisory board for Akcea/Ionis, Amgen, Eli Lilly, F. Hoffman LaRoche, Merck, Pfizer, and Sanofi/ Regeneron Pharmaceuticals, Inc. Christopher P. Cannon has received research grants from Accumetrics, Arisaph, AstraZeneca, Boehringer Ingelheim, GlaxoSmithKline, Janssen, Merck, Takeda, Amgen, BMS, American College of Cardiology, and Sanofi/Regeneron Pharmaceuticals, Inc; and is a consultant and on the advisory board for Boehringer Ingelheim CSL Behring, Essentialis, GlaxoSmithKline, Merck, Kowa, Takeda, BMS, Pfizer, Sanofi, Regeneron Pharmaceuticals, Inc., and Lipimedix. Alexia Letierce is an employee and a stockholder of Sanofi. Maja Bujas-Bobanovic is an employee and a stockholder of Sanofi. Marie T. BaccaraDinet is an employee and a stockholder of Sanofi. Michael J. Louie is an employee and stockholder of Regeneron Pharmaceuticals, Inc. Naveed Sattar has received honoraria from Merck; is a consultant and on the advisory board for Sanofi, Amgen, Eli Lilly, Boehringer Ingelheim, AstraZeneca, and Merck; and has been involved in the conduct of the GAUSS3 clinical trial of PCSK9 inhibition in patients with reported statin intolerance, sponsored by Amgen. Helen M. Colhoun reports grants, personal fees, and non-financial support from Sanofi and Regeneron Pharmaceuticals, Inc., during the conduct of the study; grants, personal fees and non-financial support from Eli Lilly \& Company, grants and other from Roche Pharmaceuticals, grants from Pfizer Inc.,
Boehringer Ingelheim, and AstraZeneca LP, and shareholding in Bayer and Roche.

Compliance with Ethics Guidelines. All study protocols were approved by the appropriate institutional review boards, and all participants provided informed, written consent. All trials were performed in accordance with the ethical principles that have their origin in the Declaration of Helsinki and all applicable amendments laid down by the World Medical Assemblies and the International Conference Harmonisation guidelines for Good Clinical Practice.

Open Access. This article is distributed under the terms of the Creative Commons Attribution-NonCommercial 4.0 International License (http://creativecommons.org/licenses/ by-nc/4.0/), which permits any noncommercial use, distribution, and reproduction in any medium, provided you give appropriate credit to the original author(s) and the source, provide a link to the Creative Commons license, and indicate if changes were made.

\section{REFERENCES}

1. Catapano AL, Graham I, De Backer G, et al. 2016 ESC/EAS guidelines for the management of dyslipidaemias: the Task Force for the Management of Dyslipidaemias of the European Society of Cardiology (ESC) and European Atherosclerosis Society (EAS) developed with the special contribution of the European Association for Cardiovascular Prevention \& Rehabilitation (EACPR). Atherosclerosis. 2016;253:281-344.

2. Authors/Task Force Members, Ryden L, Grant PJ, et al. ESC guidelines on diabetes, pre-diabetes, and cardiovascular diseases developed in collaboration with the EASD: the Task Force on diabetes, pre-diabetes, and cardiovascular diseases of the European Society of Cardiology (ESC) and developed in collaboration with the European Association for the Study of Diabetes (EASD). Eur Heart J. 2013;34(39):3035-87.

3. Piepoli MF, Hoes AW, Agewall S, et al. 2016 European guidelines on cardiovascular disease prevention in clinical practice: the Sixth Joint Task Force of the European Society of Cardiology and Other Societies on Cardiovascular Disease Prevention in 
Clinical Practice (constituted by representatives of 10 societies and by invited experts). Developed with the special contribution of the European Association for Cardiovascular Prevention \& Rehabilitation (EACPR). Eur Heart J. 2016;37(29):2315-81.

4. Sarwar N, Gao P, Seshasai SR, et al. Diabetes mellitus, fasting blood glucose concentration, and risk of vascular disease: a collaborative meta-analysis of 102 prospective studies. Lancet. 2010;375(9733):2215-22.

5. Jacobson TA, Ito MK, Maki KC, et al. National Lipid Association recommendations for patient-centered management of dyslipidemia: part 1-full report. J Clin Lipidol. 2015;9(2):129-69.

6. Lloyd-Jones DM, Morris PB, Ballantyne CM, et al. 2017 focused update of the 2016 ACC expert consensus decision pathway on the role of non-statin therapies for LDL-cholesterol lowering in the management of atherosclerotic cardiovascular disease risk: a report of the American College of Cardiology Task Force on expert consensus decision pathways. J Am Coll Cardiol. 2017;70(14):1785-822.

7. Garber AJ, Abrahamson MJ, Barzilay JI, et al. American Association of Clinical Endocrinologists' comprehensive diabetes management algorithm 2013 consensus statement-executive summary. Endocr Pract. 2013;19(3):536-57.

8. American Diabetes Association. Standards of medical care in diabetes-2018. Diabetes Care. 2018;41(Suppl. 1):S1-159.

9. Leiter LA, Lundman P, da Silva PM, Drexel H, Junger C, Gitt AK. Persistent lipid abnormalities in statin-treated patients with diabetes mellitus in Europe and Canada: results of the Dyslipidaemia International Study. Diabet Med. 2011;28(11):1343-51.

10. Wong ND, Zhao Y, Patel R, et al. Cardiovascular risk factor targets and cardiovascular disease event risk in diabetes: a pooling project of the atherosclerosis risk in communities study, multi-ethnic study of atherosclerosis, and Jackson Heart Study. Diabetes Care. 2016;39(5):668-76.

11. Landmesser U, Chapman MJ, Stock JK, et al. Update of ESC/EAS Task Force on practical clinical guidance for proprotein convertase subtilisin/kexin type 9 inhibition in patients with atherosclerotic cardiovascular disease or in familial hypercholesterolaemia. Eur Heart J. 2017;39:1131-43.

12. Krauss RM. Lipids and lipoproteins in patients with type 2 diabetes. Diabetes Care. 2004;27(6):1496-504.
13. Taskinen MR, Boren J. New insights into the pathophysiology of dyslipidemia in type 2 diabetes. Atherosclerosis. 2015;239(2):483-95.

14. Leiter LA, Cariou B, Muller-Wieland D, et al. Efficacy and safety of alirocumab in insulin-treated individuals with type 1 or type 2 diabetes and high cardiovascular risk: the ODYSSEY DM-INSULIN randomized trial. Diabetes Obes Metab. 2017;19(12):1781-92.

15. Ray KK, Leiter LA, Muller-Wieland D, et al. Alirocumab vs usual lipid-lowering care as add-on to statin therapy in individuals with type 2 diabetes and mixed dyslipidaemia: the ODYSSEY DM-DYSLIPIDEMIA randomized trial. Diabetes Obes Metab. 2018. https://doi.org/10.1111/dom.13257.

16. Robinson JG, Farnier M, Krempf M, et al. Efficacy and safety of alirocumab in reducing lipids and cardiovascular events. $\mathrm{N}$ Engl J Med. 2015;372(16):1489-99.

17. Ginsberg HN, Rader DJ, Raal FJ, et al. Efficacy and safety of alirocumab in patients with heterozygous familial hypercholesterolemia and LDL-C of $160 \mathrm{mg} / \mathrm{dl}$ or higher. Cardiovasc Drugs Ther. 2016;30(5):473-83.

18. Kereiakes DJ, Robinson JG, Cannon CP, et al. Efficacy and safety of the proprotein convertase subtilisin/kexin type 9 inhibitor alirocumab among high cardiovascular risk patients on maximally tolerated statin therapy: the ODYSSEY COMBO I study. Am Heart J. 2015;169(6):906-15.e13.

19. Kastelein JJ, Ginsberg HN, Langslet G, et al. ODYSSEY FH I and FH II: 78 week results with alirocumab treatment in 735 patients with heterozygous familial hypercholesterolaemia. Eur Heart J. 2015;36(43):2996-3003.

20. Roth EM, Taskinen MR, Ginsberg HN, et al. Monotherapy with the PCSK9 inhibitor alirocumab versus ezetimibe in patients with hypercholesterolemia: results of a 24 week, double-blind, randomized phase 3 trial. Int $\mathrm{J}$ Cardiol. 2014;176:55-61.

21. Climent E, Pérez-Calahorra S, Marco-Benedí V, et al. Effect of LDL cholesterol, statins and presence of mutations on the prevalence of type 2 diabetes in heterozygous familial hypercholesterolemia. Sci Rep. 2017; 7:5596.

22. Besseling J, Kastelein JJ, Defesche JC, Hutten BA, Hovingh GK. Association between familial hypercholesterolemia and prevalence of type 2 diabetes mellitus. JAMA. 2015;313(10):1029-36.

23. Roth EM, Bujas-Bobanovic M, Louie MJ, Cariou B. Patient and physician perspectives on mode of 
administration of the PCSK9 monoclonal antibody alirocumab, an injectable medication to lower LDLC levels. Clin Ther. 2015;37(9):1945-54.e6.

24. Schwartz GG, Bessac L, Berdan LG, et al. Effect of alirocumab, a monoclonal antibody to PCSK9, on long-term cardiovascular outcomes following acute coronary syndromes: rationale and design of the ODYSSEY outcomes trial. Am Heart J. 2014;168(5):682-9.

25. Colhoun HM, Ginsberg HN, Robinson JG, et al. No effect of PCSK9 inhibitor alirocumab on the incidence of diabetes in a pooled analysis from 10 ODYSSEY Phase 3 studies. Eur Heart J. 2016;37(39):2981-9.

26. Swerdlow DI, Preiss D, Kuchenbaecker KB, et al. HMG-coenzyme A reductase inhibition, type 2 diabetes, and bodyweight: evidence from genetic analysis and randomised trials. Lancet. 2015;385(9965):351-61.

27. Ference BA, Robinson JG, Brook RD, et al. Variation in PCSK9 and HMGCR and risk of cardiovascular disease and diabetes. $\mathrm{N}$ Engl J Med. 2016;375(22):2144-53.

28. Lotta LA, Sharp SJ, Burgess S, et al. Association between low-density lipoprotein cholesterol-lowering genetic variants and risk of type 2 diabetes: a meta-analysis. JAMA. 2016;316(13):1383-91.

29. Sabatine MS, Leiter LA, Wiviott SD, et al. Cardiovascular safety and efficacy of the PCSK9 inhibitor evolocumab in patients with and without diabetes and the effect of evolocumab on glycaemia and risk of new-onset diabetes: a prespecified analysis of the FOURIER randomised controlled trial. Lancet Diabetes Endocrinol. 2017;5(12):941-50.

30. de Carvalho LSF, Campos AM, Sposito AC. Proprotein convertase subtilisin/kexin type 9 (PCSK9) inhibitors and incident type 2 diabetes: a systematic review and meta-analysis with Over 96,000 patientyears. Diabetes Care. 2018;41(2):364-7.

31. Farnier M, Gaudet D, Valcheva V, Minini P, Miller $\mathrm{K}$, Cariou B. Efficacy of alirocumab in high cardiovascular risk populations with or without heterozygous familial hypercholesterolemia: pooled analysis of eight ODYSSEY Phase 3 clinical program trials. Int J Cardiol. 2016;223:750-7.

32. Cannon CP, Cariou B, Blom D, et al. Efficacy and safety of alirocumab in high cardiovascular risk patients with inadequately controlled hypercholesterolaemia on maximally tolerated doses of statins: the ODYSSEY COMBO II randomized controlled trial. Eur Heart J. 2015;36(19):1186-94.

33. Bays H, Gaudet D, Weiss R, et al. Alirocumab as addon to atorvastatin versus other lipid treatment strategies: ODYSSEY OPTIONS I randomized trial. J Clin Endocrinol Metab. 2015;100(8):3140-8.

34. Sattar N, Preiss D, Robinson JG, et al. Lipid-lowering efficacy of the PCSK9 inhibitor evolocumab (AMG 145 ) in patients with type 2 diabetes: a meta-analysis of individual patient data. Lancet Diabetes Endocrinol. 2016;4(5):403-10. 